\title{
An Alternative Motivation for the Indirect Approach to Closed-loop Identification
}

\author{
Lennart Ljung and Urban Forssell \\ Department of Electrical Engineering \\ Linkping University, S-581 83 Linkping, Sweden \\ WWW: http://www. control.isy.liu.se \\ Email: ljung@isy.liu.se, ufo@isy.liu.se
}

1997-12-01

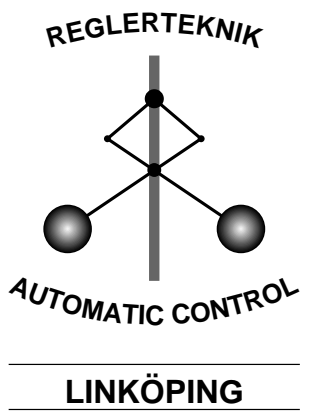

Report no.: LiTH-ISY-R-1989

Submitted to IEEE Transactions on Automatic Control.

Technical reports from the Automatic Control group in Linkping are available by anonymous ftp at the address 130.236.20.24 (ftp.control.isy.liu.se). This report is contained in the compressed postscript file $1989 \cdot \mathrm{ps} . \mathrm{Z}$. 


\title{
An Alternative Motivation for the Indirect Approach to Closed-loop Identification
}

\author{
Lennart Ljung and Urban Forssell*
}

December 4, 1997

\begin{abstract}
Direct prediction error identification of systems operating in closed loop may lead to biased results due to the correlation between the input and the output noise. We study this error, what factors affect it and how it may be avoided. In particular, the role of the noise model is discussed and we show how the noise model should be parameterized to avoid the bias. Apart from giving important insights into the properties of the direct method, this provides a nonstandard motivation for the indirect method.
\end{abstract}

\section{Introduction}

When performing identification experiments on unstable systems it is necessary to do this in closedloop with a stabilizing controller. Other reasons for considering closed-loop identification might be that the system has to be controlled for production economic or safety reasons, or that the intended model use is model-based control design. The latter case is also known as 'identification for control' or controlrelevant identification and has been a hot topic for some time now [1-5].

The interest in closed-loop identification is not new, however, and various aspects of this problem have been studied since the mid 60's, see, e.g., [69]. The text books $[10,11]$ also contain material

${ }^{*}$ L. Ljung and U. Forssell are both with the Division of Automatic Control, Department of Electrical Engineering, Linköping University, S-581 83 Linköping, Sweden. Email: ljung@isy.liu.se,ufo@isy.liu.se. on closed-loop identification. A recent account of available methods and their properties can be found in [12].

Depending on what assumptions are made about the feedback one may distinguish between three main classes of closed-loop identification methods $[7,11$, 12]: direct, indirect, and joint input-output methods.

The direct method consists in applying a prediction error method directly to measured input-out data, ignoring possible feedback. This approach should be regarded as the first choice of methods for closedloop identification. The main reasons for this are the following. The direct method gives consistency and optimal accuracy, given that the true noise characteristics are correctly modeled. It can be applied to systems with arbitrary feedback mechanisms and require no special software (other than that used for standard open-loop identification). A drawback is that we need good noise models. In practice this means that we must include a sufficiently flexible, parameterized noise model (which out-rules output error models). In case a fixed, or too "small", noise model is used the results will be biased. The reason for this bias error is that there is correlation between the output noise and the input. This is also why other methods, like instrumental variables, spectral analysis and subspace methods, fail when applied directly to closed-loop data.

For the indirect method to be applicable the feedback structure must be known (and linear), and it is also required that an external reference signal is used and that this measurable (alt. the input must be measurable and the feedback perfectly known and noise free). Under these circumstances one can pro- 
ceed as follows to identify the open-loop system. First one should identify the closed-loop system using measurements of the reference and the output. Note that this is an "open-loop problem" if the reference signal and the output additive noise are uncorrelated, which typically is the case. Hence any identification method that works in open loop can be applied in this first step. As a second step one should then compute an estimate of the open-loop system using the knowledge of the controller. Since the last step in this method may be performed in a multitude of ways, there are a number of alternative forms of this method available today. Here we will limit the study to just one special formulation of this general closed-loop identification method that effectively utilizes that in prediction error methods the parameterization may be arbitrary. More precisely, we will use a model of the closed-loop system that is parameterized in terms of the open-loop system parameters in the first step of the method. This will immediately give us an estimate of the open-loop system and thus make the second step superfluous. This idea was apparently first mentioned as an exercise in [10]. We stress that this method gives unbiased results even when applied with fixed/erroneous noise models, since we have transformed the closed-loop identification problem into an open-loop one.

In the following we will review some results that characterizes the bias error in case of direct prediction error identification and as a side-result we will see that the only way that this error can be avoided, in general, is by using the indirect method. For a more comprehensive study of the problems and possibilities with closed-loop identification see [12].

\section{Some Basics in Prediction Error Identification}

In prediction error identification one typically considers linear model structures parameterized in terms of a parameter vector $\theta$ :

$$
y(t)=G(q, \theta) u(t)+H(q, \theta) e(t)
$$

Here $G(q, \theta)$ and $H(q, \theta)$ are rational functions of $q^{-1}$, the unit delay operator $\left(q^{-1} u(t)=u(t-1)\right.$, etc. $)$ parameterized in terms of $\theta, H(q, \theta)$ monic; $y(t)$ is the output; $u(t)$ is the input; $e(t)$ is white noise.

In standard least-squares prediction error identification one finds the parameter estimates through numerical minimization of a criterion function of the form

$$
V_{N}(\theta)=\frac{1}{N} \sum_{t=1}^{N} \varepsilon^{2}(t, \theta)
$$

Here $\varepsilon(t, \theta)$ are the prediction errors, which for the model (1) can be expressed as

$$
\varepsilon(t, \theta)=H^{-1}(q, \theta)(y(t)-G(q, \theta) u(t))
$$

Under general conditions, see [10], it can be shown that the estimate will converge to the minimizing argument of the average function

$$
\bar{V}(\theta)=\frac{1}{2 \pi} \int_{-\pi}^{\pi} \Phi_{\varepsilon}(\omega) d \omega
$$

where $\Phi_{\varepsilon}(\omega)$ is the power spectral density of $\varepsilon(t, \theta)$.

It is also possible to consider pre-filtering to tune the identification criterion to give smaller errors in certain frequency bands. Suppose a stable pre-filter $L(q)$ is used, then the filtered prediction errors are $\varepsilon_{F}(t, \theta)=L(q) H^{-1}(q, \theta)(y(t)-G(q, \theta) u(t))$ and the limiting estimate will the minimize the integral of $\Phi_{\varepsilon_{F}}(\omega)$. Note that any effect of pre-filtering can be included in the noise model $H(q, \theta)$, hence we will only discuss the case $L(q) \equiv 1$ in the sequel.

\section{Assumed Output Feedback Set-up}

In this section we will specify the assumptions we make on the system to be identified.

The "true" system is given by

$$
y(t)=G_{0}(q) u(t)+v(t), v(t)=H_{0}(q) e_{0}(t)
$$

where $H_{0}(q)$ is monic and inversely stable and $\left\{e_{0}(t)\right\}$ is a sequence of independent random variables, with zero mean and variance $\lambda_{0}$. The identification experiments are performed with the feedback regulator

$$
u(t)=r(t)-F_{y}(q) y(t)
$$


controlling the plant. Here $\{r(t)\}$ is an external reference signal, independent of $\left\{e_{0}(t)\right\} ; F_{y}(q)$ is a linear controller chosen so that either $G_{0}(q)$ or $F_{y}(q)$ contains a delay, in order to ensure that the closed-loop system is well posed.

With the definitions (5) and (6), the closed-loop system can be written

$$
y(t)=G_{0}(q) S_{0}(q) r(t)+S_{0}(q) v(t)
$$

where $S_{0}(q)$ denotes the sensitivity function,

$$
S_{0}(q)=\frac{1}{1+G_{0}(q) F_{y}(q)}
$$

The input becomes

$$
u(t)=S_{0}(q) r(t)-F_{y}(q) S_{0}(q) v(t)
$$

Here we may note that there will always be a nonzero contribution from the noise $v(t)$ to the input, given by the second term in expression (9), unless $F_{y}(q)=0$ (open-loop operation).

\section{Analysis of the Bias Error: Part I}

Suppose now that the system (5), controlled using the feedback law (6), is to be identified using a model structure of the form (1). For simplicity of the argument we for the time being assume that $G$ and $H$ are independently parameterized. Using (7) and (9) we may write the prediction errors (3) as

$$
\begin{aligned}
\varepsilon(t, \theta)=H^{-1} & (q, \theta)\left[\left(G_{0}(q)-G(q, \theta)\right) S_{0}(q) r(t)+\right. \\
& \left.+\left(1+G(q, \theta) F_{y}(q)\right) S_{0}(q) v(t)\right]
\end{aligned}
$$

The spectrum is (dropping the arguments $e^{i \omega}$ and $\theta$ )

$\Phi_{\varepsilon}(\omega)=\left|G_{0}-G\right|^{2} \frac{\left|S_{0}\right|^{2} \Phi_{r}(\omega)}{|H|^{2}}+\frac{\left|1+G F_{y}\right|^{2}\left|S_{0}\right|^{2} \Phi_{v}(\omega)}{|H|^{2}}$

Recall that the in the limit, as $N \rightarrow \infty$, the prediction error estimate will minimize the integral of $\Phi_{\varepsilon}$ :

$$
\theta_{o p t}=\arg \min _{\theta} \int_{-\pi}^{\pi} \Phi_{\varepsilon}(\omega) d \omega
$$

We denote the resulting optimal transfer functions $G_{\text {opt }}$ and $H_{\text {opt }}\left(G_{o p t}(q)=G\left(q, \theta_{\text {opt }}\right)\right.$ etc. $)$.

What will $G_{\text {opt }}$ be then? Well, from (11) it is clear that the optimal $G$ will be a compromise between making the first term, $\left|G_{0}-G\right|^{2}$, and the second term, $\left|1+G F_{y}\right|^{2}$, small. Thus, in general, the resulting optimal model $G_{o p t}$ will not be equal to the true system $G_{0}$, i.e., there will be a bias error. Before quantifying this bias error in terms of the reference and noise spectra and the noise model used, let us stop and think about if, and how, this bias error can be avoided.

\section{Interlude: How to Avoid the Bias Error - The Indirect Method}

The basic problem in (11) is that both the first and the second term depend on $G$, an ideal situation would have been that the second term was $G$ independent (as in the open-loop case).

Suppose that the noise model is parameterized as

$$
H(q, \theta)=\bar{H}(q, \theta)\left(1+G(q, \theta) F_{y}(q)\right)
$$

where the parameters in $\bar{H}(q, \theta)$ are independent of the ones in $G(q, \theta)$. The spectrum of the prediction errors then becomes

$$
\Phi_{\varepsilon}(\omega)=\frac{\left|G_{0}-G\right|^{2}}{\left|1+G F_{y}\right|^{2}} \frac{\left|S_{0}\right|^{2} \Phi_{r}(\omega)}{|\bar{H}|^{2}}+\frac{\left|S_{0}\right|^{2} \Phi_{v}(\omega)}{|\bar{H}|^{2}}
$$

and we see that $G$ only enters in the first term, as desired. Moreover, it should also be clear that this is the only way to make the second term in (11) $G$ independent.

Note that in $(13), \bar{H}(q, \theta)$ may be arbitrary. In particular, when we only are interested in identifying the system dynamics we may choose $\bar{H}$ to be fixed, $\bar{H}(q, \theta)=H_{*}(q)$, so that

$$
H(q, \theta)=H_{*}(q)\left(1+G(q, \theta) F_{y}(q)\right)
$$

This will have the effect that the expression for the limiting $G$-model becomes (with some abuse of nota- 
tion)

$$
G_{\text {opt }}=\arg \min _{G} \int_{-\pi}^{\pi} \frac{\left|G_{0}-G\right|^{2}}{\left|1+G F_{y}\right|^{2}} \frac{\left|S_{0}\right|^{2} \Phi_{r}(\omega)}{\left|H_{*}\right|^{2}} d \omega
$$

Now if the parameterization is sufficiently flexible, so that the true system is in the model set, then we will always have that $G_{o p t}=G_{0}$ (i.e., no bias), even if the noise model $H_{*}(q)$ is incorrect.

What that does then the special parameterization (15) mean? First of all, it is important to note that the prediction error theory is parameterizationindependent. Here we have used this fact to parameterize the noise model in terms of the dynamics model. There is nothing strange with that, so do ARX, ARMAX models. Second, if we compute the predictor for the model (1) with $H$ parameterized as in (15) and using that $u(t)=r(t)-F_{y}(q) y(t)$ we get

$\hat{y}(t \mid \theta)=H^{-1}(q, \theta) G(q, \theta) u(t)+\left(1-H^{-1}(q, \theta)\right) y(t)$

$$
=H_{*}^{-1}(q, \theta) \frac{G(q, \theta)}{1+G(q, \theta)} r(t)+\left(1-H_{*}^{-1}(q, \theta)\right) y
$$

But this is exactly the predictor for the model of the closed-loop system

$$
y(t)=\frac{G(q, \theta)}{1+G(q, \theta)} r(t)+H_{*}(q) e(t)
$$

which in turn is a smart parameterization of the indirect method which avoids the sometimes difficult second step in this method.

The conclusion is that if we want to be sure that we do not have a bias error in the direct method we should use the special parameterization (15), i.e., we should use the indirect method (19). We believe this provides new insight into both the direct and the indirect methods as far as the role of the parameterizations of and connections between the two methods are concerned. For related material, see, e.g., [13, 14].

\section{Analysis of the Bias Error: Part II}

In the previous section we saw that by cleverly parameterizing the noise model, the bias error can be avoided even if no effort is spent on modeling the true noise characteristics. To complete our survey of bias results for the direct method we will now state a result that was first given in [15] which will explictly show how large the bias error will be and what factors affect it.

Suppose that we want to identify the system (5) using the model (1) where the noise model is fixed, $H(q, \theta)=H_{*}(q)$ (the results to follow easily extend to the case of an independently parameterized noise model). Then the limiting model is given by $[14,15]$

$$
G_{\text {opt }}=\arg \min _{G} \int_{-\pi}^{\pi}\left|G_{0}-G-B\right|^{2} \frac{\Phi_{u}(\omega)}{\left|H_{*}\right|^{2}} d \omega
$$

where ( $B$ for bias)

$$
|B|^{2}=\frac{\lambda_{0}}{\Phi_{u}(\omega)} \frac{\left|F_{y} S_{0}\right|^{2} \Phi_{v}(\omega)}{\Phi_{u}(\omega)}\left|H_{0}-H_{*}\right|^{2}
$$

From this expression we see that if $F_{y}=0$ (open-loop $t)_{\text {operation) }}$ then the bias term $B$ will always be zero. From (21) it is also clear that the bias-inclination will be small in frequency ranges where either (or all) of the following holds:

- The noise model is good $\left(\left|H_{0}-H_{*}\right|^{2}\right.$ small).

- The part of the input spectrum stemming from the noise fed back to the input (i.e., $\left.\left|F_{y} S_{0}\right|{ }^{2} \Phi_{v}(\omega)\right)$ is small compared to the total input spectrum $\left(\Phi_{u}(\omega)\right)$.

- The signal-to-noise ratio is good $\left(\lambda_{0} / \Phi_{u}(\omega)\right.$ small).

This indicates that in many practical cases the bias error will be small. One such case is when the noise variance $\lambda_{0}$ is small compared to $\Phi_{u}(\omega)$, another is when a reasonably flexible, independently parameterized noise model is used. It is also important to remember that in practice the error in the estimated models will be due to both bias and variance errors, so the importance of the bias errors should not be over-emphasized. 


\section{Conclusions}

By studying the bias error due to feedback in the estimated transfer functions when using the direct method we have obtained a non-standard motivation for the indirect approach to closed-loop identification.

We have seen that direct identification of systems operating in closed-loop gives biased results whenever the true noise characteristics are not correctly modeled. In most cases the bias error will be small, especially if the signal-to-noise ratio is good, but the only way it can completely be removed is by using a custom parameterization of the noise model or, equivalently, by using the indirect method.

\section{References}

[1] R. J. P. Schrama, "Accurate models for control design: the necessity of an iterative scheme," IEEE Transactions on Automatic Control, vol. 37, pp. 991-994, 1992.

[2] M. Gevers, "Towards a Joint Design of Identification and Control," in Essays on Control: Perspectives in the Theory and its Applications, H. L. Trentelman and J. C. Willems, Eds., pp. 111-151. Birkhäuser, 1993.

[3] P. M. J. Van den Hof and R. J. P. Schrama, "Identification and Control - Closed-loop Issues," Automatica, vol. 31, no. 12, pp. 17511770, 1995.

[4] Z. Zang, R. R. Bitmead, and M. Gevers, "Iterative Weighted Least-squares Identification and Weighted LQG Control Design," Automatica, vol. 31, no. 11, pp. 1577-1594, 1995.

[5] W. S. Lee, B. D. O. Anderson, I. M. Y. Mareels, and R. L. Kosut, "On some key issues in the windsurfer approach to adaptive robust control," Automatica, vol. 31, no. 11, pp. 1619-1636, 1995.

[6] H. Akaike, "Some Problems in the Application of the Cross-Spectral Method," in Spectral Analysis of Time Series, B. Harris, Ed., pp. 81-107. John Wiley \& Sons, 1967.
[7] I. Gustavsson, L. Ljung, and T. Söderström, "Identification of Processes in Closed Loop Identifiability and Accuracy Aspects," Automatica, vol. 13, pp. 59-75, 1977.

[8] B.D.O. Anderson and M. Gevers, "Identifiability of linear stochastic systems operating under linear feedback," Automatica, vol. 18, no. 2, pp. 195-213, 1982.

[9] M. Gevers and L. Ljung, "Optimal experiment design with respect to the intended model application," Automatica, vol. 22, pp. 543-554, 1986.

[10] L. Ljung, System Identification: Theory for the User, Prentice-Hall, 1987.

[11] T. Söderström and P. Stoica, System Identification, Prentice-Hall International, 1989.

[12] U. Forssell, Properties and Usage of Closed-loop Identification Methods, Licentiate thesis LIUTEK-LIC-1997:42, Department of Electrical Engineering, Linköping University, Linköping, Sweden, September 1997.

[13] B. Egardt, "On the role of noise models for approximate closed loop identification," in Proceedings of the European Control Conference, Brussels, Belgium, 1997.

[14] L. Ljung, "Identification in closed loop: some aspects on direct and indirect approaches," in Preprints of the 11th IFAC Symposium on System Identification, Fukuoka, Japan, July 1997, vol. 1, pp. 141-146.

[15] L. Ljung, "Information contents in identification data from closed loop operation," in Proc. 32nd IEEE Conference on Decision and Control, San Antonio, TX, December 1993, pp. 2242-2247. 